\title{
Osteosyntheseversagen bei Patellafrakturen
}

\author{
Jürgen Degreif
}

\section{Zusammenfassung}

Die Patellafraktur gehört mit vergleichsweise klaren Behandlungsrichtlinien nicht zu den Problemfrakturen der unfallchirurgischen Fachliteratur. Dies steht jedoch im Widerspruch zur hohen Rate von Komplikationen und Osteosyntheseversagen. In der vorliegenden Arbeit sollen einige Kontroversen der Therapie diskutiert und die wichtigsten Gründe für fehl- geschlagene Osteosynthesen anhand zweier aussagekräftiger Fallbeispiele herausgearbeitet werden. Eine technisch perfekte Osteosynthese ist gerade bei Problemfrakturen eine absolut notwendige, aber nicht hinreichende Bedingung für die Frakturheilung. Bei sehr schwierigen Ausgangssituationen bleibt mitunter lediglich die Patellektomie, wobei nach Möglichkeit der Teilpatellektomie der Vorzug zu geben ist.

\section{Einleitung}

Die Patellafraktur gehört zu den vermeintlich gelösten Frakturen und unfallchirurgischen Standards. Die Klassifikation unterscheidet nicht dislozierte Frakturen, Querfrakturen, obere oder untere Polfrakturen, Längsfrakturen und Trümmerfrakturen. Die dislozierte Querfraktur ist die häufigste Frakturform und wird typischerweise mittels Drahtzuggurtung stabilisiert. Zusammen mit der Olekranonfraktur ist die Patellafraktur das klassische Lehrbeispiel für die Zuggurtungsversorgung in den entsprechenden Grundkursen der Operationslehre. Insofern scheinen kaum Unklarheiten zu bestehen.

Bezeichnenderweise beschäftigen sich auch wenige Publikationen und Fachvorträge der letzten Jahre mit der Patellafraktur. Lediglich die Vorstellung neuer Osteosyntheseverfahren wie spezielle Nagelungsverfahren gibt Anlass zu publikatorischem Fleiß bezüglich der Patellafraktur [6 ]. Diese neuen Verfahren spielen bislang jedoch eine Außenseiterrolle.

OP-JOURNAL 2004; 20: 210-216

(c) Georg Thieme Verlag KG Stuttgart · New York
Die klassische Zuggurtung mit oder ohne zusätzlicher Cerclage stellt nach wie vor den goldenen Behandlungsstandard dar.

Angesichts beachtlicher Komplikationsraten in der Literatur $[7,11,16]$ drängt sich die Frage auf, inwieweit beim Behandlungsalgorhythmus der Patellafraktur tatsächlich alle Fragen beantwortet sind und was die Gründe für die vergleichsweise häufigen Osteosyntheseversagen sind.

So wurden in der eigenen Klinik in den letzten 3 Jahren 21 Patellafrakturen osteosynthetisch versorgt. In 5 Fällen kam es zum revisionspflichtigen Osteosyntheseversagen, davon in 2 Fällen sogar mehrfach (Abb.1 und 2). In der vorliegenden Übersicht sollen einerseits Kontroversen der Frakturversorgung diskutiert und andererseits die wichtigsten Ursachen für Osteosyntheseversager anhand von Fallbeispielen vorgestellt und Lösungsvorschläge gemacht werden.

Vorausgesetzt werden grundlegende Kenntnisse bezüglich Unfallhergang, Biomechanik des Streckapparates mit der Patella als Hypomochlion, Diagnostik, Prinzipien der Zuggurtung und der frühfunktionellen Nachbehandlung. Auch die unspezifischen Komplikationen wie Erguss, Serom, Hämatom, Infekt, Wundrandnekrose sowie Thrombose und Embolie bedürfen keiner besonderen Betrachtung, sondern werden nach den allgemein gültigen Grundsätzen behandelt. Die speziellen Komplikationen wie Weichteilirritation durch vorstehende Drähte, verzögerte Frakturheilung und Osteosyntheseversagen sind dagegen $\mathrm{Ge}$ genstand dieser Arbeit.

\section{Hauptteil}

Fragen und Kontroversen

Bezüglich einer Reihe von Aspekten (Tab.1) besteht immer noch Diskussionsbedarf. Erfolgte die Frakturversorgung früher routinemäßig über einen querverlaufenden Hautschnitt in den Streckfalten der Haut, so hat sich in den letzten Jahren die längsverlaufende Schnittführung durchgesetzt. Die guten Erfahrungen aus der Knieprothetik zeigen, dass die frühere Furcht vor Narbenkontrakturen nach längsverlaufendem Schnitt unbegründet ist. Die Frakturreposition ist genau so gut möglich und das Bohren der Kirschner-Drähte einfacher. Zeigte nach querverlaufender Schnittführung der distale Wundrand gelegentlich eine Durchblutungsminderung, so ist jetzt der gesamte Wundrand zu beiden Seiten gleichmäßig gut durchblutet und für später eventuell notwendige Prothetik kann derselbe Schnitt wieder benutzt werden.

Bei den häufigen Querfrakturen muss nach der klassischen Zuggurtungsosteosynthese mit zwei 1,8 oder $2,0 \mathrm{~mm}$ Kirschner-Drähten und einem $1,25 \mathrm{~mm}$ Cerclagedraht darauf geachtet werden, dass dieser Cerclagedraht dem Knochen exakt anliegt [13].

Eine zusätzliche zirkuläre Cerclage ist aus Gründen der Knochendurchblutung nicht notwendig und bleibt besonderen Situationen wie Mehrfragment- und Trümmerbrüchen sowie der Revisionschirurgie vorbehalten. 


\section{Tab. 1 Fragen und Kontroversen}

- Schnittführung quer oder längs

- Zuggurtung mit oder ohne zusätzliche Zerklage

- Indikation zur Patellektomie

- Postoperative Ruhigstellung

\section{Tab. 2 Gründe für fehlgeschlagene} Osteosynthesen

Hochgradig instabile Fraktur

(Trümmerfraktur)

Fehlerhafte Operationstechnik

Vorgeschädigter Knochen

Entnahme eines Kreuzbandtrans-

plantates

Osteoporose

Unkooperativer Patient

Demenz

Morbus Parkinson

Alkoholismus

Übertriebene, zu aktive Übungs-

behandlung

Die Indikation zur partiellen oder gar totalen Patellektomie bei Mehrfragmentund Trümmerfrakturen gehört zu den schwierigsten Fragen [2] und ist nicht pauschal zu beantworten, zumal die diesbezüglichen Behandlungsergebnisse in der Literatur differieren. So wird von den Befürwortern der totalen Patellektomie der Vorteil der kürzeren Immobilisation und der komplikationsärmeren Operationstechnik sowie die frühzeitige Wiederaufnahme der Berufstätigkeit angeführt $[5,10]$. Entsprechende Spätergebnisse mit lediglich $20 \%$ schlechten Resultaten [8] scheinen dieses Vorgehen zu bestätigen. Andererseits werden von Wilkinson [17] 40\% schlechte Resultate und von Burton [4] 65\% mäßige bis schlechte Resultate berichtet. Der Erhalt von Teilen der Patella, vor allem der proximalen Hälfte bzw. der proximalen 2/3 scheint hier von Vorteil zu sein, um die Hypomochlionfunktion der Patella zu erhalten $[1,3,9,12,13,14,15]$. Bei aussichtslosen Trümmersituationen kann im Einzelfall auch eine primäre Patellektomie sinnvoll sein. Es ist jedoch ausdrücklich in das Ermessen des erstbehandelnden Chirurgen gestellt, ob er eine primäre Osteosynthese und damit einen Erhaltungsversuch der Patella durchführt, wohl wissend, dass beim Scheitern der Osteosynthese eine sekundäre Teil- oder totale Patellektomie erforderlich sein kann.
Bei primär übungsstabiler Osteosynthese wird eine frühfunktionelle, gipsfreie Nachbehandlung gefordert [3]. Dies ist auch gültig unter der Voraussetzung, dass der Verletzte das Bein nur in absoluter Streckstellung belastet und Bewegungsübungen nur ohne Muskelanspannung durchführt. Nach meiner Erfahrung sind viele Patienten mit diesem Konzept überfordert, so dass es sich bewährt hat, eine abnehmbare Schiene in Streckstellung anzulegen und Bewegungsübungen unter Abnahme dieser Schiene nur unter krankengymnastischer Aufsicht durchführen zu lassen. Bei unkooperativen Patienten ist dieses Konzept entsprechend strenger zu handhaben.

\section{Osteosyntheseversagen}

Anhand zweier eklatanter Fallbeispiele sollen die verschiedenen Ursachen (Tab.2) für ein Scheitern der Osteosynthese erläutert werden.

Im ersten Fallbeispiel (Abb.1) hatte sich ein zum Unfallzeitpunkt 37-jähriger Mann durch ein direktes adäquates Trauma eine Patellamehrfragmentfraktur mit drei Hauptfragmenten zugezogen (Abb.1a,b). Erschwerend kam hinzu, dass die Patella durch die 3 Monate zuvor durchgeführte Entnahme eines BoneTendon-Bone-Kreuzbandersatzes vorgeschädigt und geschwächt war. Die Primärversorgung erfolgte durch eine Zuggurtungsosteosynthese (Abb.1 c, d). Nach zunächst komplikationslosem Verlauf zeigt sich nach 4 Monaten im Röntgenbild eine Dehiszens der Fragmente (Abb.1e). Es erfolgt die operative Revision mit Neuanlage der Zuggurtungsosteosynthese, diesmal unter Verwendung eines geflochtenen Stahlseiles als Zuggurtungsdraht und mit zusätzlicher Spongiosaplastik (Abb.1f,g). Auch diesmal war der Verlauf zunächst komplikationsfrei. Das Osteosyntheseversagen zeigt sich nach nunmehr 5 Monaten mit einer erneuten Dehiszens als Zeichen der ausgebliebenen Frakturheilung (Abb.1 h).

Die erneute Revision erfolgt unter Verwendung zweier Zugschrauben statt der zuvor verwandten Kirschner-Drähte sowie erneuter Spongiosaplastik. Zur Anregung der Knochenheilung wurde ein handelsübliches BMP-Präparat (Ossacur $^{\circledR}$ ) in den Frakturspalt eingebracht und die Nachbehandlung erfolgte deutlich restriktiver als zuvor mit Ruhigstellung in Streckstellung und Übungsbehandlung lediglich bis $40^{\circ}$ Beugestellung.
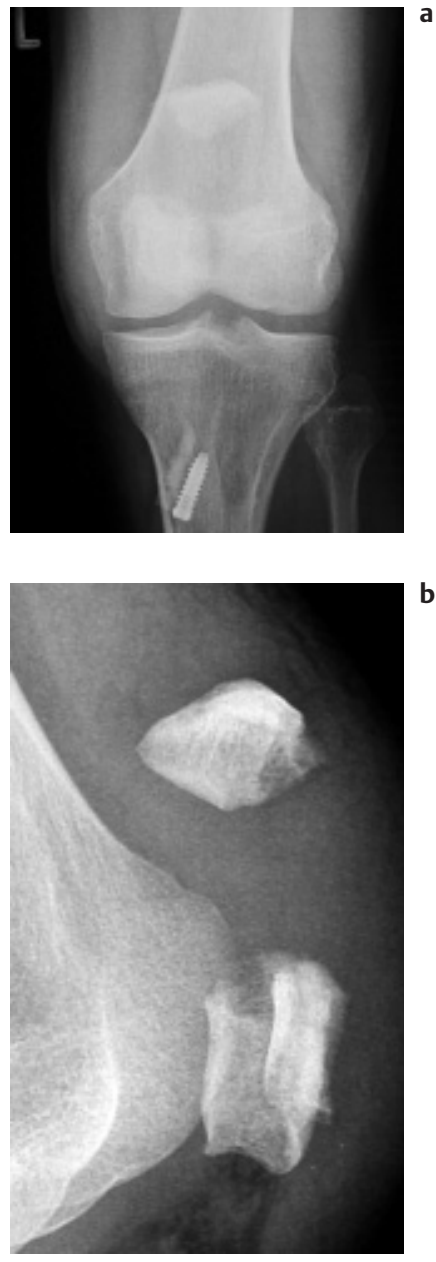

Abb.1au.b Mehrfragmentfraktur eines 37-jährigen Mannes, bei dem 3 Monate zuvor ein BoneTendon-Bone-Transplantat zum Kreuzbandersatz entnommen worden war.

Es kam in der Folge zur Ausheilung der Fraktur (Abb.1 i,j).

\section{Kritische Wertung:}

Lediglich 3 Monate nach vorausgegangener Kreuzbandplastik mit Entnahme eines Knochenblocks aus der distalen Patella muss man sowohl eine Vitalitätsstörung als auch eine mechanische Schwächung annehmen. Darüber hinaus ist es bei dem erneuten adäquaten Trauma zu einer Mehrfragmentfraktur mit entsprechendem Anspruch an die Osteosynthesetechnik gekommen. Aus diesem Grund ist bereits primär zusätzlich zur Zuggurtung eine Cerclage angelegt worden. An der Zuggurtung ist zu kritisieren, dass die Kirschner-Drähte vor allem proximal zu weit überstehen und durch Irritation der Quadrizepssehne dislozieren. Der dazugehörige achterförmige Cerclagedraht liegt dem Knochen nicht unmittelbar an 

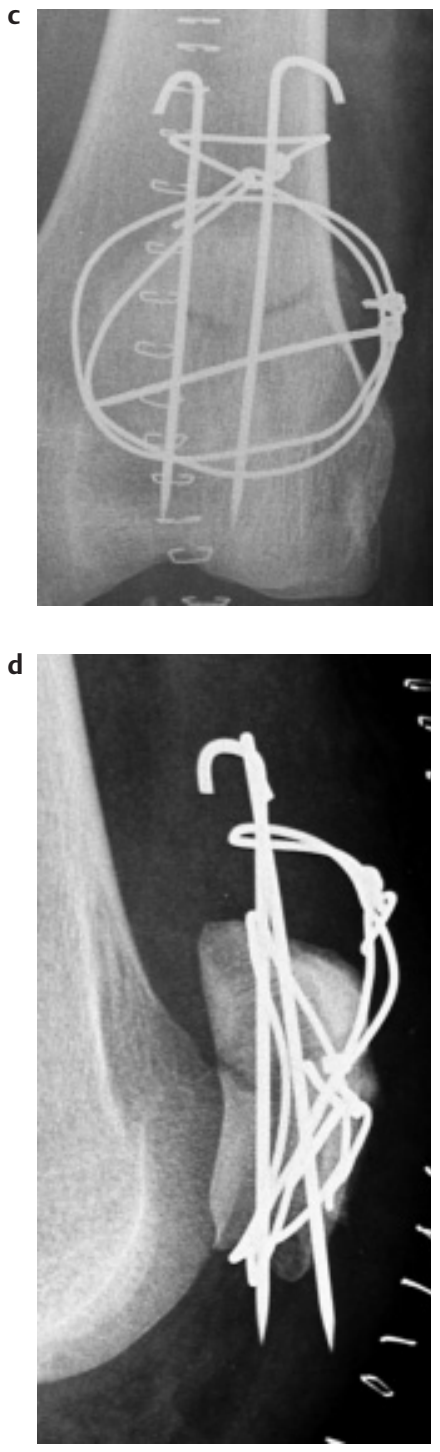

Abb.1cu.d Zuggurtungsosteosynthese + Cerclage. Die Kirschner-Drähte stehen proximal zu weit vor und der Cerclagedraht liegt vor allem proximal dem Patellapol nicht exakt an.

und kann somit seine Funktion nicht dauerhaft erfüllen. Durch den zirkulären Cerclagedraht ist allerdings eine Mindeststabilität gewährleistet. So ist es auch zu verstehen, dass dieses Konstrukt immerhin 4 Monate gehalten hat bis es zur Dehiszens kam. Bei einer unkomplizierten Querfraktur ohne die genannte Vitalitätsstörung und vorbestehende Stabilitätsminderung wäre die Fraktur in dieser Zeit wahrscheinlich trotz der mangelhaften Osteosynthese geheilt.

Gerade die Erfahrung, dass auch suboptimale Osteosynthesen erfolgreich sein können, verleitet mitunter dazu, intraoperativ Fehler zu akzeptieren! e

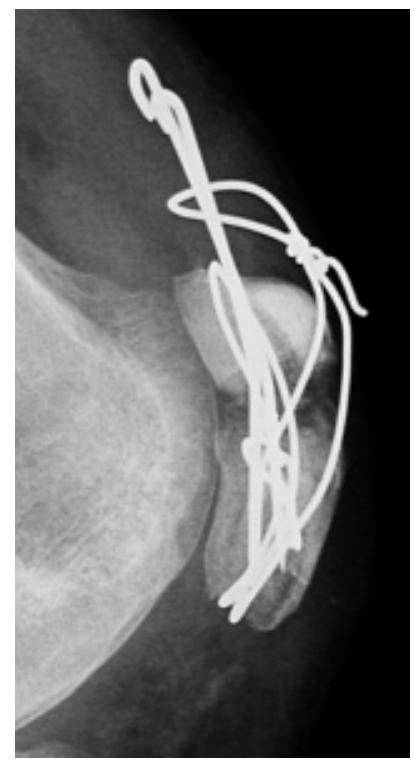

Abb.1e Erstes Osteosyntheseversagen nach 4 Monaten.
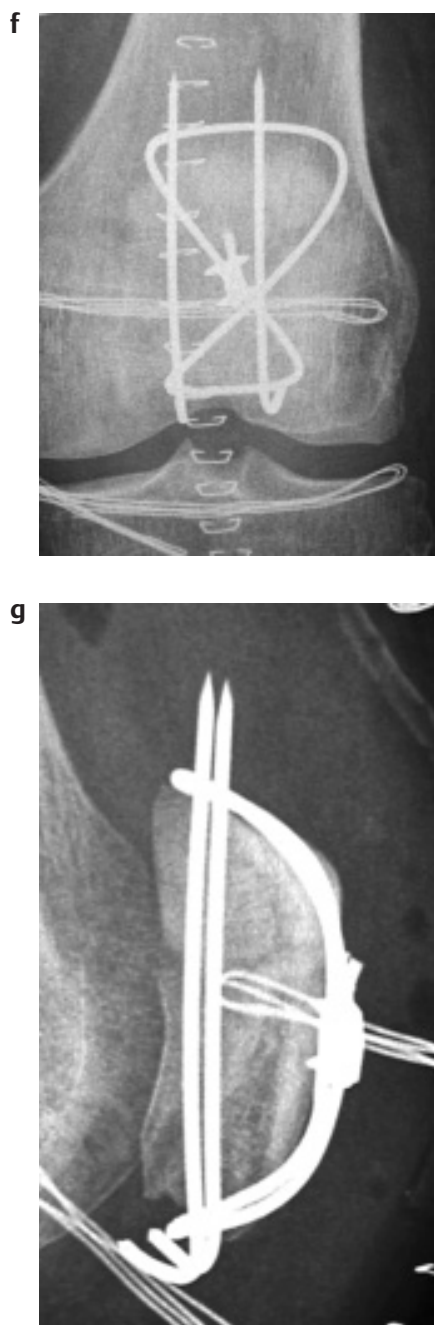

Abb.1fu.g Erneute Zuggurtungsosteosynthese mittels eines geflochtenen Stahlseiles und zusätzlicher Spongiosaplastik.

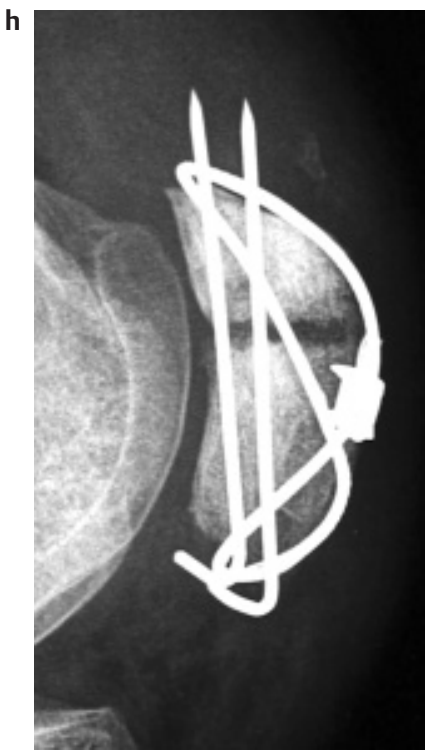

Abb.1h Zweites Osteosyntheseversagen 5 Monate nach der Revisions-OP.
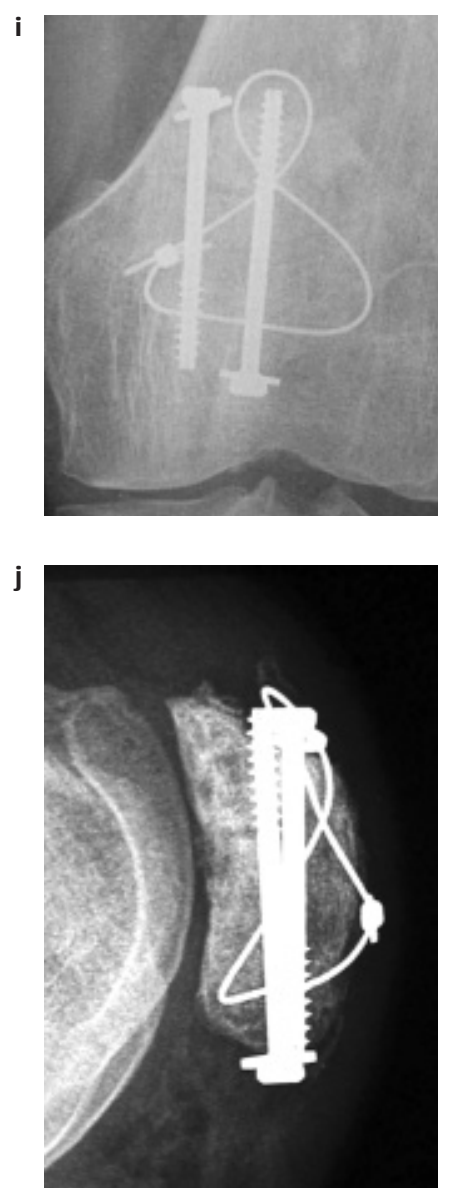

Abb.1iu.j Ausheilungsbilder nach erneuter Osteosynthese mittels Zugschrauben + Drahtcerclage sowie erneuter Spongiosaplastik und Einbringen eines BMPPräparates. 
Bei der Revision zeigte sich, dass Teile der Trümmerfraktur in der Tat geheilt waren und es fanden sich nunmehr 2 Hauptfragmente. Demgemäß wurde eine erneute Zuggurtung in korrekter Technik angelegt mit korrekt gekürzten Kirschner-Drähten und perfekt am Knochen anliegender Cerclage, für die aus Stabilitätsgründen ein geflochtenes Stahlseil hergenommen wurde, das sich dem Knochen sehr schön anlegt, eine hohe Reißfestigkeit besitzt und sich perfekt spannen lässt. In der Folgezeit legte der Patient wie schon in der ersten postoperativen Phase einen sehr großen Übungsfleiß an den Tag und wollte möglichst rasch wieder die volle Beweglichkeit erreichen. Auch dies ist ein Umstand, der zumindest teilweise den komplizierten Verlauf verursacht hat. Das Scheitern dieser zweiten, diesmal technisch nicht zu beanstandenden Osteosynthese erfolgte nach 5 Monaten und ist einerseits auf den übertriebenen Übungsfleiß des Patienten und andererseits die bereits genannte Vitalitätsstörung des Knochens zurückzuführen.

Für die jetzt insgesamt 3. Osteosynthese wurden statt der Kirschner-Drähte Kleinfragmentzugschrauben hergenommen und zusätzlich eine achterförmige Drahtcerclage angelegt. Kombiniert wurde dieses Vorgehen mit einer erneuten Spongiosaplastik, diesmal mit Zusatz eines handelsüblichen BMP-Präparates. Das Nachbehandlungskonzept war jetzt sehr streng und wurde vom Patienten auch akzeptiert mit Ruhigstellung des Kniegelenkes in einer abnehmbaren Schiene in Streckstellung und Übungsbehandlung unter Vermeidung von Muskelanspannungen limitiert auf eine Beugung von $40^{\circ}$. Unter all diesen Voraussetzungen heilte die Fraktur bzw. Pseudarthrose jetzt aus.

Anlässlich dieser Publikation wurde der Patient nach mittlerweile 2 Jahren nach der letzten Operation kontaktiert. Er ist beschwerdefrei und startet in seiner Freizeit bei Halbmarathonläufen. Als Ursachen für den komplizierten Verlauf und die mehrfach fehlgeschlagenen Osteosynthesen sind folgende Gründe zu nennen:

- Mechanisch und biologisch vorgeschädigte Patella nach Entnahme eines Kreuzbandtransplantates.

- Technisch mangelhafte Erstosteosynthese.

- Zu aktives Nachbehandlungskonzept.

Beim zweiten exemplarischen Fall (Abb.2) handelt es sich um eine zum Un-

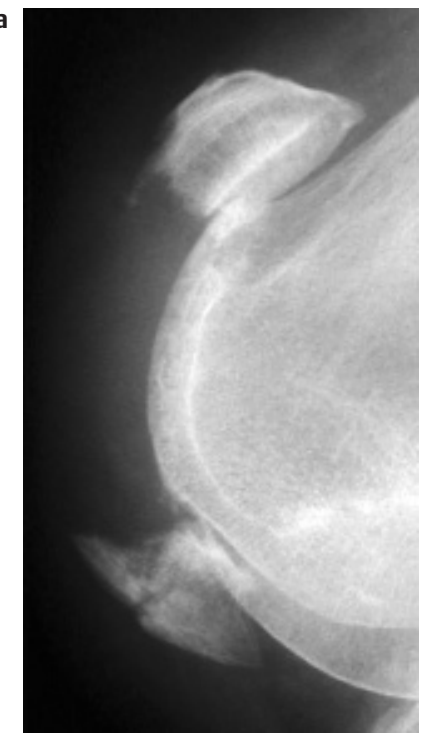

Abb.2a Trümmerfraktur vor allem des unteren Patellapoles einer 77-jährigen Frau mit Demenz.

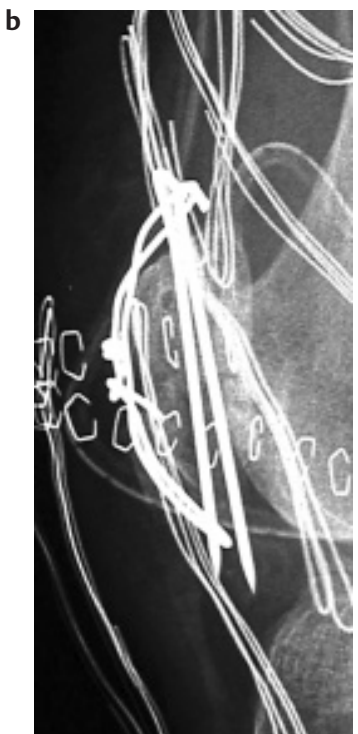

Abb. 2b Zuggurtungsosteosynthese. Ein Kirschner-Draht ist distal nicht vom Cerclagedraht gefasst.

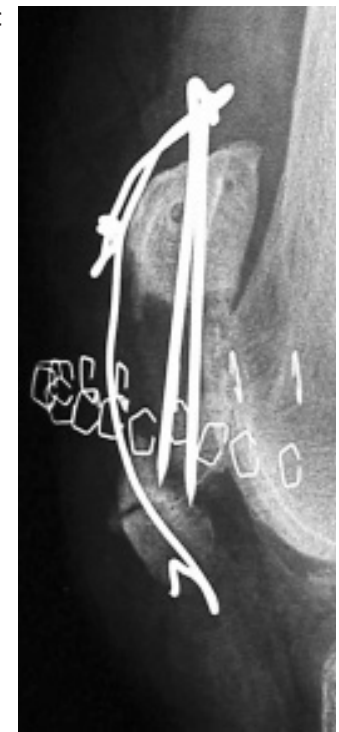

Abb.2c Erstes Osteosyntheseversagen nach Sturz aus dem Bett.
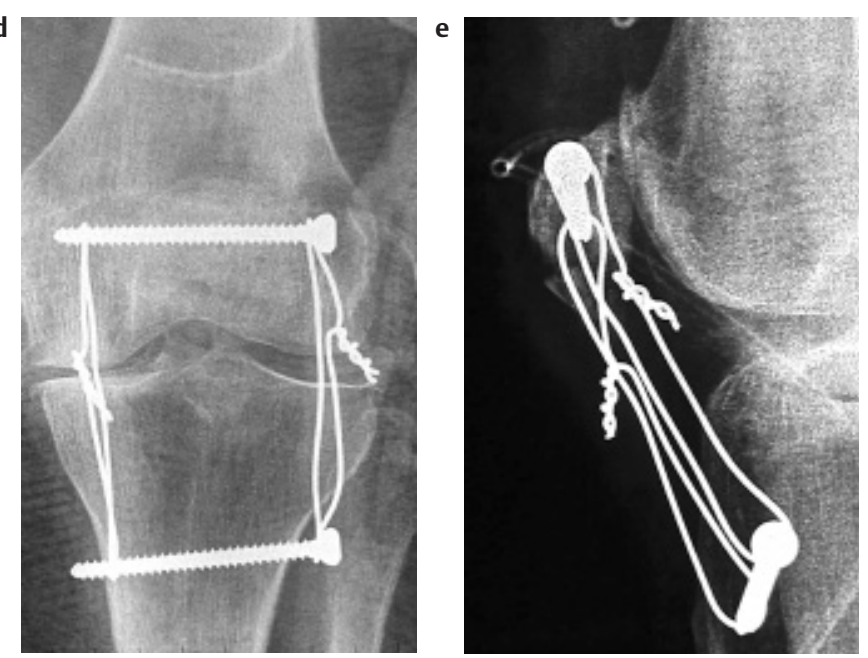

Abb.2du.e Partielle Patellektomie am distalen Pol mit transossärer Ligamentum-patellae-Refixation an das proximale Hauptfragment und Rahmencerclage zur Tuberositas tibiae als Augmentation.

fallzeitpunkt 77-jährige Frau mit bekannter beginnender Demenz und Mehrfragmentsituation im Bereich des unteren Patellapoles (Abb.2a). Auf der ersten postoperativen Kontrollaufnahme (Abb.2b) erkennt man, dass der 2. Kirschner-Draht distal von dem Cerclagedraht nicht gefasst wird, so dass auch hier keine korrekte Osteosynthesetechnik anzunehmen ist.

Nach wenigen Tagen war die Patientin beim Versuch, alleine aus dem Bett aufzu- stehen, gestürzt und die Röntgenkontrolle (Abb. 2 c) zeigt den geöffneten Cerclagedraht mit weiter Dehiszens der Fragmente. Schon bei der Erstversorgung war die Fraktursituation angesichts der Trümmerfraktur und des osteoporotisch weichen Knochens vom Operateur als extrem schwierig bezeichnet worden, so dass jetzt bei der Revision ein erneuter Osteosyntheseversuch aussichtslos war und eine partielle Patellektomie im distalen Bereich durchgeführt wurde. Ein Hauptfragment mit Ansätzen des Liga- 

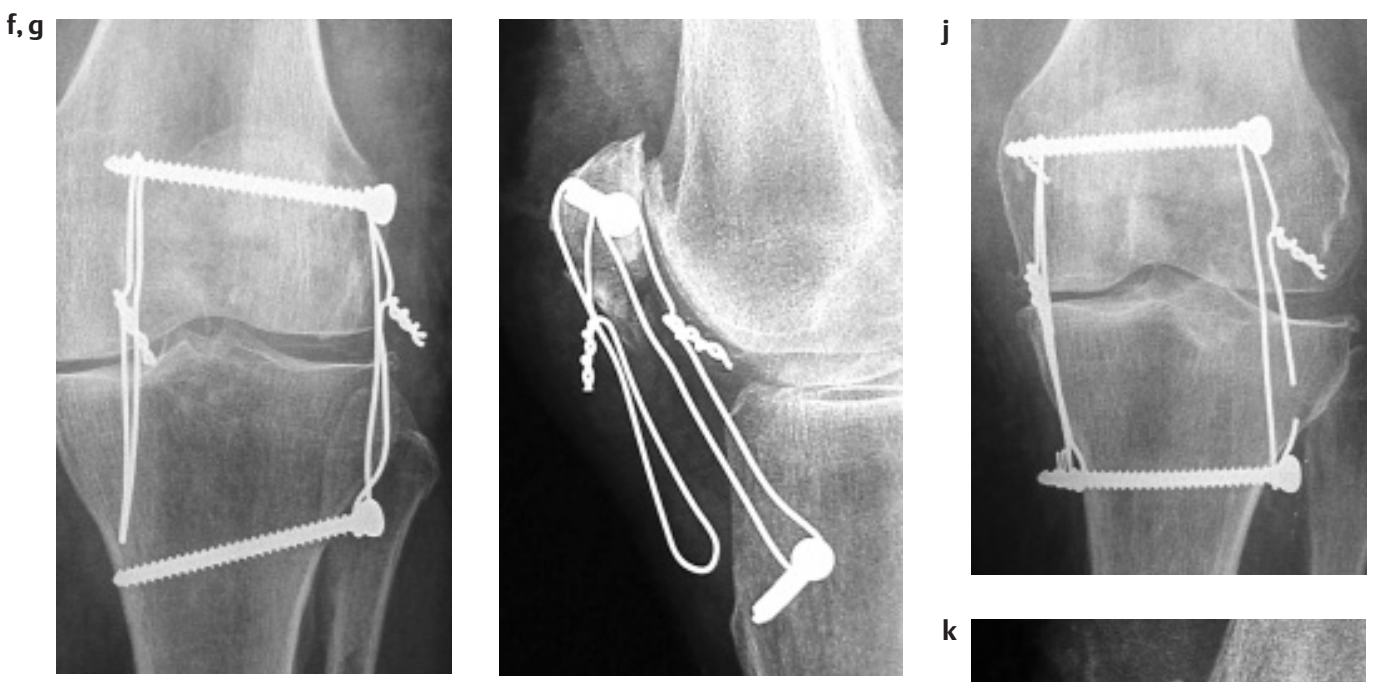

Abb.2fu.g Dislokation des medialen Drahtes.
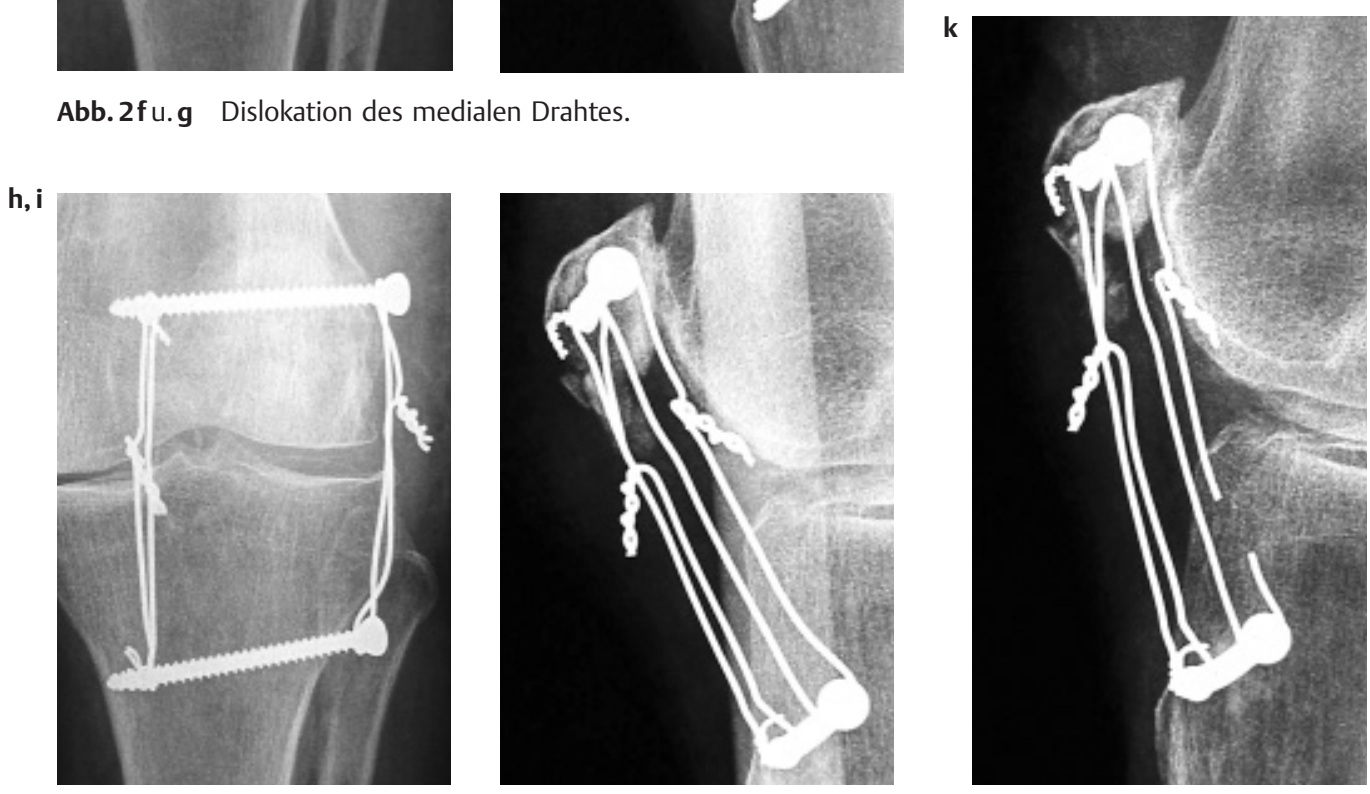

Abb. $\mathbf{2 h u . i \quad R e p o s i t i o n ~ u n d ~ S i c h e r u n g ~ d e s ~ D r a h t e s . ~}$

Abb.2ju.k Bruch des lateralen Drahtes.

mentum patellae wurde mittels transossärer Nähte zum proximalen Hauptfragment hin adaptiert und diese keinesfalls übungs- oder gar belastungsstabile Situation durch eine Rahmencerclage zur Tuberositas tibiae hin, wie wir sie auch bei Ligamentum-patellae-Rupturen durchführen, gesichert (Abb. 2 d,e). Damit lässt sich die Spannung des M. quadrizeps femoris suffizient aufnehmen. Beim Anziehen der Cerclagedrähte kann es leicht zu einer Überdistraktion des relaxierten Muskels im Sinne eines Patellatiefstandes kommen. Dies lässt sich leicht vermeiden, indem man sich die Position der kontralateralen Kniescheibe mit dem C-Bogen als Vergleich einstellt.

Wiederum wenige Tage später wurde in einer Routineverlaufskontrolle eine Dislokation des medialen Drahtes aus der Schraubenaufhängung heraus festgestellt (Abb.2f,g). Diese Komplikation wäre bei einem transossär durch einen Bohrkanal geführten Draht natürlich nicht möglich. Andererseits gewährleistet die Schraube eine gleichmäßigere Krafteinleitung in den Knochen und vermeidet gerade beim osteoporotischen Knochen ein seitliches Einschneiden des Cerclagedrahtes im Bereich der Bohrloch-Ein- und -Austrittsstellen. Bei der Revision wurde somit der Draht lediglich wieder eingehängt und gegen eine erneute Dislokation gesichert (Abb. $\mathbf{2}$ h,i).

4 Wochen später zeigt sich in der Verlaufskontrolle ein Bruch des lateralen Cerclagedrahtes (Abb.2j, k). An dieser Stelle ist anzumerken, dass die Patientin infolge ihrer vorbestehenden Demenz in keinster Weise kooperativ war. Schienen zur Ruhigstellung wurden sehr rasch von ihr selbst wieder abgenommen, so dass man von einer frühzeitigen Vollbelastungssituation ausgehen muss.
Auf einen geschlossenen Gipsverband wurde aus Furcht vor Hautschäden unsererseits verzichtet. Auffällig bei diesem mittlerweile dritten Osteosyntheseversagen ist der Bruch des Drahtes an einer mechanisch eher weniger beanspruchten Stelle. Ich hätte den Bruch eher an der Biegestelle an einer der beiden Schrauben oder im Bereich des Drahtzwirbels erwartet. Da wir nach 4 Wochen noch keine hinreichende Stabilität des Streckapparates befürchteten, wurde der gebrochene Draht ausgetauscht gegen ein geflochtenes Stahlseil (Abb.21, m).

Die nächsten Kontrollen (Abb.2n,o) zeigen die Situation zum Zeitpunkt der geplanten ME. Mittlerweile 12 Wochen nach der Erstversorgung konnte die Patientin mit dieser Situation gehen und das Knie gegen Widerstand strecken, somit wurde von weiteren rekonstruktiven Maßnahmen Abstand genommen und le- 


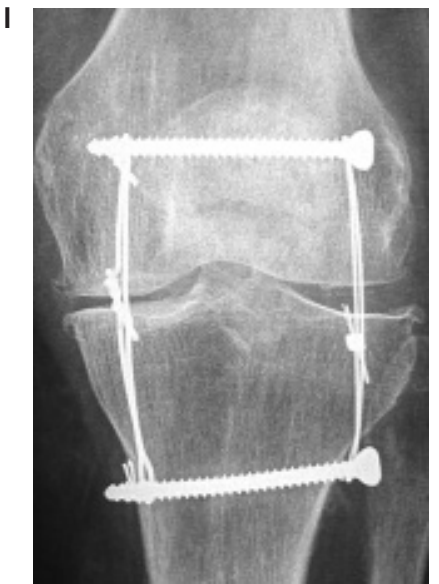

m

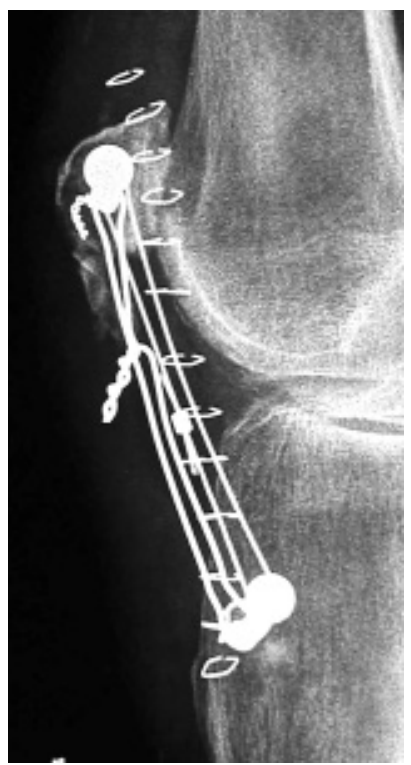

Abb.2lu.m Austausch des gebrochenen Drahtes gegen ein geflochtenes Stahlseil. n
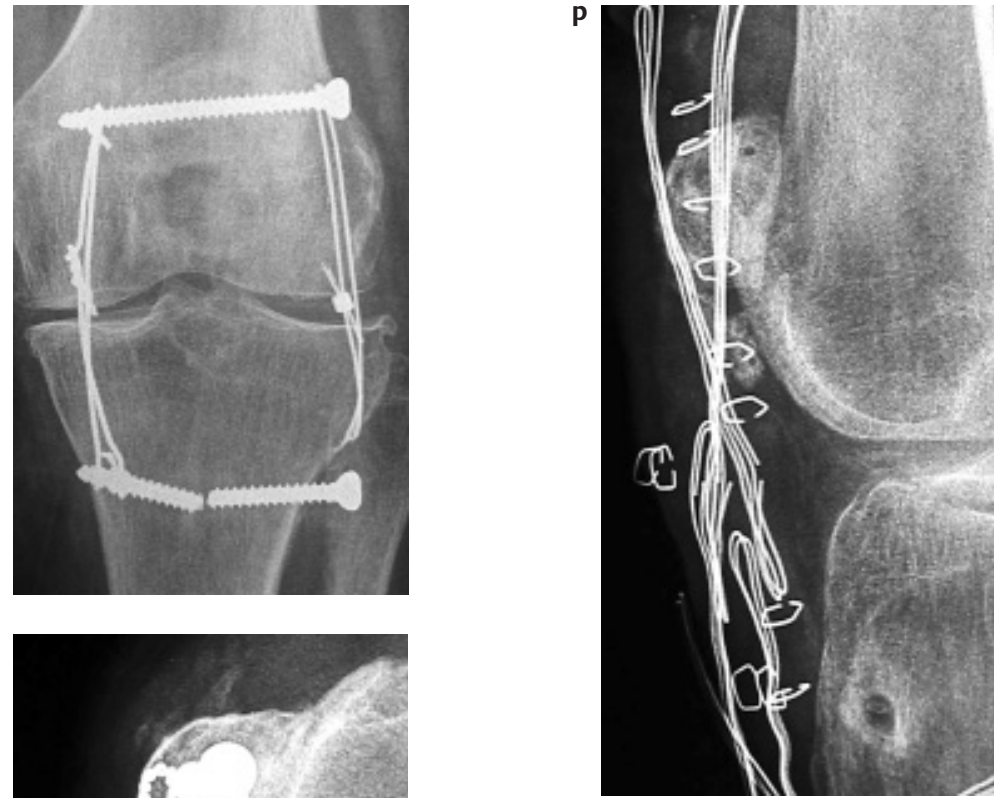

Abb.2p Gesamtmetallentfernung.

Dennoch ist gerade bei schwierigen Ausgangssituationen eine perfekte Operationstechnik eine notwendige, aber leider nicht immer hinreichende Bedingung für die Frakturheilung.

Bei schwierigen bzw. sehr instabilen Ausgangssituationen kann statt der üblichen K-Drähte eine Zugschraubenosteosynthese mit der gewohnten Cerclage kombiniert werden (Abb.1i u.j.j). Die Indikation zur Patellektomie ist streng zu stellen. Meist lassen sich zumindest proximale Anteile und damit die Hypomochlionfunktion der Patella erhalten.

Abb. 2nu.o 12 Wochen nach der Erstversorgung Riss beider Cerclagedrähte und Bruch der distalen Schraube.

\section{Literatur}

${ }^{1}$ Andrews JR, Hughston JC. Treatment of Patellar Fractures by Partial Patellectomy. South Med J 1977; 70: 809

um beide Kirschner-Drähte herumgeführt wurde. Die Aufhängung der Drahtaugmentation für die Ligamentum-patellae-Naht ist ein grundsätzlich probates Verfahren. Dabei sollten allerdings die Drähte gegen ein Luxieren gesichert werden.

\section{Schlussfolgerung}

Zusammenfassend stellen Patellafrakturen den Unfallchirurgen immer wieder vor schwierige Aufgaben mit häufig fehlgeschlagenenen Osteosynthesen. Die Gründe (Tab. 2) sind nicht immer in einer fehlerhaften Osteosynthesetechnik zu finden.

(a) Ochsner PE. Indikationen und Kontraindikationen der Patellektomie. Z Or-

thop 1986; 124: 266

${ }^{3}$ Bühren V, Trentz O, Henneberger G. Die operative Behandlung der Patellafraktur. Chirurg 1989; 60: 723

${ }^{4}$ Burton VW. Results of Excision of the Patella.

Surg Gynecol Obstet 1972; 135: 753

${ }^{5}$ Duthie HL, Hutchinson JR. The Results of Partial and Total Excision of the Patella. J Bone Joint Surg 1958; 40B: 75

${ }^{6}$ Gehr J, Friedl W. Probleme der Zuggurtungsosteosynthese von Patellafrakturen und deren Konsequenzen für weitere Implantatentwicklungen Der XS-Nagel Chirurg 2001; 72 Nr 11: 1309

${ }^{7}$ Holz U, Thielemann FW, Zahedi B. Biomechanik, Operationstechnik und Ergebnisse der Patellafrakturen. Hefte Unfallheilkd 1975; 212: 142 
${ }^{8}$ Jakobsen J, Christensen KS, Rasmussen OS Patellectomy-a 20-year Follow-up. Acta Orthop Scand 1985; 56: 430

${ }^{9}$ Jaskulka R, Ittner G, Raffezeder U. Die chirurgische Versorgung dislozierter Patellafrakturen - Therapie und Ergebnisse. Unfallchirurgie 1989; 15: 253

${ }^{10}$ Levack B, Flannagan JP, Hobbs S. Results of Surgical Treatment of Patellar Fracture. J Bone Joint Surg 1985; 67B: 416

${ }^{11}$ Neumann HS, Winckler S, Strobel M. Langzeitergebnisse nach operativer Versorgung von Patellafrakturen Unfallchirurg 1993; 96: 305

${ }^{12}$ Pandey AK, Pandey S, Pandey P. Results of partial patellectomy. Arch Orthop Trauma Surg 1991; 110: 246
${ }^{13}$ Rogge D, Oestern H-J, Grosse F. Die Patellafraktur. Orthopäde 1981; 14: 266

${ }^{14}$ Sutton FS, Thompson CH, Lipke J, Kettlekamp DB. The Effect of Patellectomy on Knee Function. J Bone Joint Surg 1976; 58A: 537

${ }^{15}$ Villiger KJ. Partielle Patellektomie. Chirurg 1970; $41: 236$

${ }^{16}$ Wendl K, Zinser R, Hochstein P. Frakturen der Kniescheibe: Eine häufig unterschätzte Verletzung Trauma und Berufskrankheit 2002; $4 \mathrm{Nr} 1: 30$

${ }^{17}$ Wilkinson J. Fractures of the Patella Treated by Total Excision. J Bone Joint Surg 1977; 59B: 352

\section{Buchbesprechung}

\section{Miller M.D., Howard R.F., Plancher K.D. Operationsatlas Sportorthopädie - Sporttraumatologie Urban \& Fischer, München, Buchneuerscheinung}

Im Juli 2004 erschien der Operationsatlas Sportorthopädie - Sporttraumatologie von Miller, Howard und Plancher in deutscher Erstauflage mit 542 Seiten und 1000 s/w sowie 500 farbigen Abbildungen. Die Presseinformation des Urban \& Fischer Verlags macht sehr neugierig auf dieses Buch: „Fundiertes Hintergrundwissen über die Gelenktraumatologie, detaillierte Beschreibungen von Eingriffen bei traumatischen oder degenerativen Belastungsschäden und Verletzungen, die Darstellung modernster bildgebender Techniken und Therapien, exzel- lentes Bildmaterial, ein schneller Überblick durch einheitliche Systematik und Kapitelaufbau“, letztlich unmittelbar in die Praxis umsetzbare Empfehlungen würden den Leser erwarten. Der formulierte Anspruch ist hoch. Die Gliederung erfolgt in den Teil 1 „Untere Extremitäten“, er enthält die Abschnitte I. Knie, II. Bein, Sprunggelenk und Fuß, III. Oberschenkel Hüfte und Becken sowie in den Teil 2 „Obere Extremitäten“ mit den Abschnitten IV. Schulter, V. Ellenbogen sowie VI. Handgelenk und Hand. Mehr als 120 der insgesamt 542 Seiten sind dem Kapital 1 „Kniearthroskopie“, demgegenüber drei Seiten dem Thema Patellarsehnen- und Quadrizepssehnenrupturen, dreieinhalb Seiten der Achillessehnenruptur und zehn Seiten dem gesamten Komplex der sportbedingten
Prof. Dr. med. Jürgen Degreif Chefarzt

der Klinik für Unfallchirurgie und Orthopädie

Städt. Kliniken Esslingen

Hirschlandstr. 97

D-73730 Esslingen
Frakturen der unteren Extremitäten gewidmet. Der als Zielgruppe angegebene Student oder junge Arzt wird den Anspruch auf Vollständigkeit, eine Systematik und Didaktik vermissen. Der Reiz dieses Buches liegt vielmehr darin, dem AOgeprägten Unfallchirurgien und Orthopäden Teile seines Fachgebietes einmal aus einem ihm weniger bekannten, nämlich dem nordamerikanisch geprägten Blickwinkel vorzustellen. Das bietet neben einem über weite Strecken erfrischenden Lesegenuss immer wieder Anlass, eigene Strategien kritisch zu hinterfragen. Ob dies den Anschaffungspreis von 165,00 Euro rechtfertigt, mag jeder für sich entscheiden.

\section{A. Luther, Erfurt}

